\title{
Serum parameters in the spectrum of coeliac disease: beyond standard antibody testing - a cohort study
}

\author{
Greetje J Tack ${ }^{1 *}$, Roy L J van Wanrooij ${ }^{1}$, B Mary E Von Blomberg ${ }^{2}$, Hedayat Amini ${ }^{2}$, Veerle M H Coupe ${ }^{3}$, \\ Petra Bonnet ${ }^{2}$, Chris J J Mulder ${ }^{1}$ and Marco W J Schreurs ${ }^{2,4}$
}

\begin{abstract}
Background: Invasive techniques are still required to distinguish between uncomplicated and complicated forms of $C D$.

Methods: We set out to investigate the potential use of novel serum parameters, including IL-6, IL-8, IL-17, IL-22, sCD25, sCD27, granzyme-B, sMICA and sCTLA-4 in patients diagnosed with active CD, CD on a GFD, Refractory coeliac disease (RCD) type I and II, and enteropathy associated T-cell lymphoma (EATL).

Results: In both active CD and RCDI-II elevated levels of the proinflammatory IL-8, IL-17 and SCD25 were observed. In addition, RCDII patients displayed higher serum levels of soluble granzyme-B and IL-6 in comparison to active CD patients. In contrast, no differences between RCDI and active CD or RCDIl were observed. Furthermore, EATL patients displayed higher levels of IL-6 as compared to all other groups.
\end{abstract}

Conclusions: A series of novel serum parameters reveal distinctive immunological characteristics of RCDII and EATL in comparison to uncomplicated CD and RCDI.

Keywords: Coeliac disease, Refractory coeliac disease, Enteropathy associated T-cell lymphoma, Cytokines, Immunological and biochemical parameters

\section{Background}

Coeliac disease $(\mathrm{CD})$ is a chronic immune-mediated inflammation of the small intestine caused by a permanent state of intolerance to ingested gluten proteins affecting genetically susceptible individuals. Its hallmarks, lymphocytic infiltration of the lamina propria, expansion of the intraepithelial lymphocyte (IEL) population, hyperplasia of the crypts and atrophy of the villi are mediated by the interplay between an innate and adaptive immune response against gluten [1]. The complex of deamidated gliadin peptide interacting with the HLA-DQ2 and/or DQ8 heterodimers on antigen presenting cells, is capable of activating the lamina propria T-helper lymphocytes, thereby initiating antibody production and a gluten-specific pro-inflammatory type 1 T-cell response (Th1) [1]. Th1

\footnotetext{
* Correspondence: g.tack@vumc.nl

${ }^{1}$ Department of Gastroenterology and Hepatology, VU University Medical Centre, PO Box 7057, Amsterdam 1007 MB, The Netherlands

Full list of author information is available at the end of the article
}

cells play an important role in pathogenesis of $\mathrm{CD}$ by secreting interleukin-2 (IL-2) that induces proliferation of T-lymphocytes and, in particular, by secreting the pro-inflammatory cytokine interferon-gamma (IFN- $\gamma$ ) [2]. Furthermore, recent evidence has indicated that Th17 cells play a pathogenic role in CD [3-5]. In addition, interleukin15 (IL-15) activates a cytotoxic response of intra-epithelial lymphocytes through IFN-y release and upregulation of NKG2D. In combination with epithelial stress, the NKG2D ligand MHC Class I chain-related A (MICA) is upregulated by enterocytes [6,7]. The interaction of MICA and NKG2D induces enterocyte destruction by IELs.

A gluten-free diet (GFD) results in mucosal recovery in the majority of patients, who are referred to as uncomplicated CD-patients. However, a small subset of adult onset $\mathrm{CD}$ patients fails to regain intestinal homeostasis after elimination of dietary gluten, or symptoms recur after initial response [8]. After careful evaluation of dietary compliance and exclusion of other possible disease-entities 
causing villous atrophy, these patients are diagnosed to suffer from refractory coeliac disease (RCD) [9]. RCD is considered a complicated form of $\mathrm{CD}$, and is divided into type I (RCDI), when patients lack an aberrant IEL population, or type II (RCDII) in which a substantial $(>20 \%)$ aberrant IEL population is found in the small intestinal mucosa $[10,11]$. An aggressive type of lymphoma which carries a dismal prognosis, the enteropathy-associated T-cell lymphoma (EATL), is thought to arise from this aberrant IEL population. An interesting observation is that both aberrant IELs as well as EATL cells display a cytotoxic phenotype and contain high levels of granzyme-B [12,13], which could therefore serve as a marker for complicated CD.

In consequence of a good response to immunosuppressive therapy, RCDI patients have a better prognosis than RCDII patients [14-17]. Therefore, early identification of CD patients developing RCDII and/or EATL enables early intervention, which will likely reduce morbidity and mortality.

Currently, antibodies against tissue transglutaminase (TGA), anti-endomysium (EMA) and deamidated gliadin peptides (DGPA) provide valuable and generally accepted serum parameters for the diagnosis and followup of uncomplicated CD. However, these antibodies are of no use in predicting and monitoring both types of RCD and EATL, implying that histological and flow cytometric analysis of duodenal biopsies are still required to distinguish between the uncomplicated and complicated forms of $\mathrm{CD}$. Additional serum markers could potentially provide us with a minimal-invasive, easy applicable test without the need to perform a gastro-duodenal endoscopy. In addition, immunological markers in the peripheral blood could provide more insight in the similarities and differences of the pathophysiology underlying the CD spectrum.

Therefore, in the present study we evaluated several immunological and biochemical parameters in sera from the five stages of $C D$, including active $C D$ (ACD), CD on GFD, RCDI-II and EATL, for their ability to differentiate between complicated and uncomplicated forms, and secondly, to gain insight in the pathophysiological relations between these disease entities. For this purpose, we analysed serum levels of the inflammatory cytokines IL-6, IL-8, IL-17and IL-22, the T-cell activation factors soluble (s)CD25 (IL2R-alpha) and $\mathrm{sCD} 27$, the T-cell dysregulation factor sCTLA-4, shown previously to be up-regulated in different autoimmune diseases, the cytotoxic T-cell parameter granzyme-B, and sMICA, previously shown to be associated with the presence of epithelial stress and malignancies.

\section{Methods}

A retrospective cohort study was conducted at a tertiary referral centre for coeliac disease in The Netherlands.
Patients previously diagnosed with (complicated) $C D$ in the VU medical center were identified and included in our study when stored serum samples at time of diagnosis were available. Overall, 92 blood samples collected for diagnostic purposes between 1997 and 2010 were included. Serum levels of a substantial number of immunological markers were determined in the five different subsets of $C D$. In addition, results from several biochemical parameters of this cohort at time of diagnosis were collected from the electronic patient file in our centre.

\section{Patients}

CD diagnosis was based on the ESPGHAN guidelines [18]. All patients included in the active CD group had positive EMA and/or TGA, and histological abnormalities grade III according to the modified Marsh classification consisting of intra-epithelial lymphocytosis, crypt hyperplasia and some degree of villous atrophy [19]. Furthermore, serum samples of these patients at time of an inactive phase of $\mathrm{CD}$ were collected. Remission of disease (GFD group) was defined by the disappearance of one or both CD antibodies upon a GFD, and if a gastroduodenal endoscopy with subsequent collection of biopsies was performed during follow-up, normalisation of mucosal abnormalities (Marsh 0 or I) was required. Patients included in the RCD group had persisting or recurring symptoms and small intestinal villous atrophy, despite strict adherence to a GFD for at least one year (assessed by a dietitian and negative TGA/EMA). The clinically validated cut-off value of more than $20 \%$ of the IELs expressing an aberrant phenotype (surface CD3-, but cytoplasmic $\mathrm{CD}^{+}$) as detected by flow cytometric analysis was used to distinguish RCD type I and II [10].

In total, 26 paired serum samples of $C D$ patients at time of disease activity (ACD group) and after normalisation of the CD associated antibodies upon a GFD (GFD group), and of an additional 40 patients with complicated $C D$ at diagnosis were included. The latter group consists of 12 RCDI, 16 RCDII and 12 EATL patients.

All procedures were in accordance with the regulations of the medical ethics committee, and all patients declared their informed consent to store and use their blood samples collected for regular diagnostics for further research.

\section{Serum parameters: Enzyme linked immunosorbent assay (ELISA)}

Levels of cytokines IL-6, IL-8, IL-17 and IL-22 were determined in serum using commercial ELISA kits (Pelikine-compact ${ }^{\mathrm{TM}}$, Sanquin, Amsterdam, The Netherlands), according to the manufacturer's instructions. Levels of soluble CD25 (sCD25), soluble CD27 (sCD27), soluble CTLA-4 (sCTLA-4), soluble MICA (sMICA) and 
granzyme-B were determined in serum using a specific commercial ELISA kit (Diaclone, Besancon, Cedex, France), according to the manufacturer's instructions.

\section{Biochemical parameters}

The concentration of C-reactive protein (CRP; g/L), erythrocyte sedimentation rate (ESR; $\mathrm{mm}$ per $1 \mathrm{~h}$ ), leukocyte count (WBC; 10E9/L), albumin $(\mathrm{g} / \mathrm{L})$ and haemoglobin $(\mathrm{Hb} ; \mathrm{mmol} / \mathrm{L})$ were extracted from the hospital patient file for all patients included at time of diagnosis.

\section{Statistical analysis}

Data were analysed and plotted using SPSS software (SPSS Inc. Chicago, Illinois, USA), using non-parametric tests as most variables examined in this study did not appear to be normally distributed. A Wilcoxon signedrank test was used for pairwise comparison of the variability of immunological and biochemical parameters among the ACD and GFD group. The latter groups were individually compared to the complicated forms of $\mathrm{CD}$ by using the Kruskal-Wallis non-parametric test to identify possible serum and biochemical differences in the spectrum of $\mathrm{CD}$. A receiver operating characteristic (ROC) curve was made of all significantly different parameters to represent the trade off between the false negative and false positive rates. As a considerable number of markers were determined, the level of significance was set at highly significant $(\mathrm{p}<0.001)$ and moderately significant $(0.001<\mathrm{p}<0.05)$.

\section{Results}

Table 1 shows the characteristics of the five subsets of CD. Serum samples of 26 ACD patients at time of diagnosis and after remission of disease on a GFD were included. In all patients $\mathrm{CD}$ associated antibodies reverted to negative upon a GFD, and in $62 \%(16 / 26)$ a biopsy was taken which revealed mucosal healing (Marsh 0/I) in all those evaluated. Overall, 12 RCDI, 16 RCDII and 12 EATL patients were included. Significant differences were found for cytokine profiles between the five subsets of $\mathrm{CD}$, as described in more detail below (Figure 1A-I).

\section{Active $\mathrm{CD}$ versus GFD}

Serum levels of the inflammatory chemokine IL-8 $(\mathrm{p}=0.00)$ and the T-cell activation factor $\mathrm{sCD} 25$ were higher in active $C D$ patients than in patients in remission on a GFD ( $p=0.00$, highly significant) as well as the Th-17 lineage-defined cytokine IL-17 levels $(\mathrm{p}=0.011$, moderately significant). Serum levels of IL-6, IL-22, sCD27, sMICA, granzyme-B and sCTLA-4 were not significantly elevated in the ACD group. In addition, levels of CRP, ESR, albumin and leukocyte counts were similar (data not shown).

\section{Uncomplicated $C D$ versus $R C D$}

The serum levels of IL-8, IL-17 and sCD25 in RCD type I and type II were comparable to those in the ACD group, however, significantly lower levels were observed in the GFD group. Moreover, in comparison to both ACD and GFD patients, RCDII patients showed increased levels of granzyme-B $(p=0.020 ; p=0.016$, both moderately significant) and IL-6 ( $\mathrm{p}=0.018 ; \mathrm{p}=0.024$, both moderately significant), respectively. Furthermore, serum levels of soluble CTLA-4 were lower in RCDII patients than those in remission upon a GFD $(\mathrm{p}=0.003$, moderately significant). Similar IL-22 serum levels were found in uncomplicated CD and RCDI-II. .

Comparison of the inflammatory parameters CRP, ESR and leukocyte count did not reveal significant differences between uncomplicated CD and RCDI-II. The concentration of albumin was lower in both RCD subsets when compared to ACD $(\mathrm{p}=0.003$, moderately significant $)$ and GFD ( $\mathrm{p}=0.000$, highly significant; Figure 2$)$.

\section{The RCD complex}

None of the markers tested was able to distinguish RCDII from RCDI. Moreover, no significant differences in levels of albumin or inflammatory parameters CRP, ESR and leukocyte count were observed.

\section{EATL versus $A C D$ and $R C D$}

The highest serum levels of IL- 6 were observed in the EATL group, were higher $(\mathrm{p}=0.000$, highly significant $)$ as compared to the ACD and GFD groups, as well as higher $(\mathrm{p}=0.002 ; \mathrm{p}=0.007$, moderately significant) than RCDI-II, respectively. Moreover, serum levels of IL-6 were clearly elevated in EATL over RCDII with an AUC of 0.82 [95\% CI: 0.649-0.971]. IL-6 levels in EATL patients tended to correlate $(0.45, \mathrm{p}=0.08)$ with CRP levels, but not with IL-17 levels. Furthermore, serum levels of sCD27 were decreased (moderately significant) in EATL patients as compared to all other groups, except RCDII in which (highly) significant $(\mathrm{p}=0.000)$ differences were found). Nevertheless, ROC analysis resulted in a very low AUC. In addition, similar levels of sMICA, granzyme-B and sCD25 were measured in EATL and RCDI-II. However, the serum albumin concentration in EATL patients was lower (moderately significant) than in ACD and both types of RCD (Figure 2).

\section{Discussion}

Currently, physicians are unable to predict who will develop complicated $C D$. As invasive techniques are still required to differentiate between uncomplicated and complicated forms of $\mathrm{CD}$, the purpose of the present 
Table 1 Characteristics of the different groups in the spectrum of coeliac disease

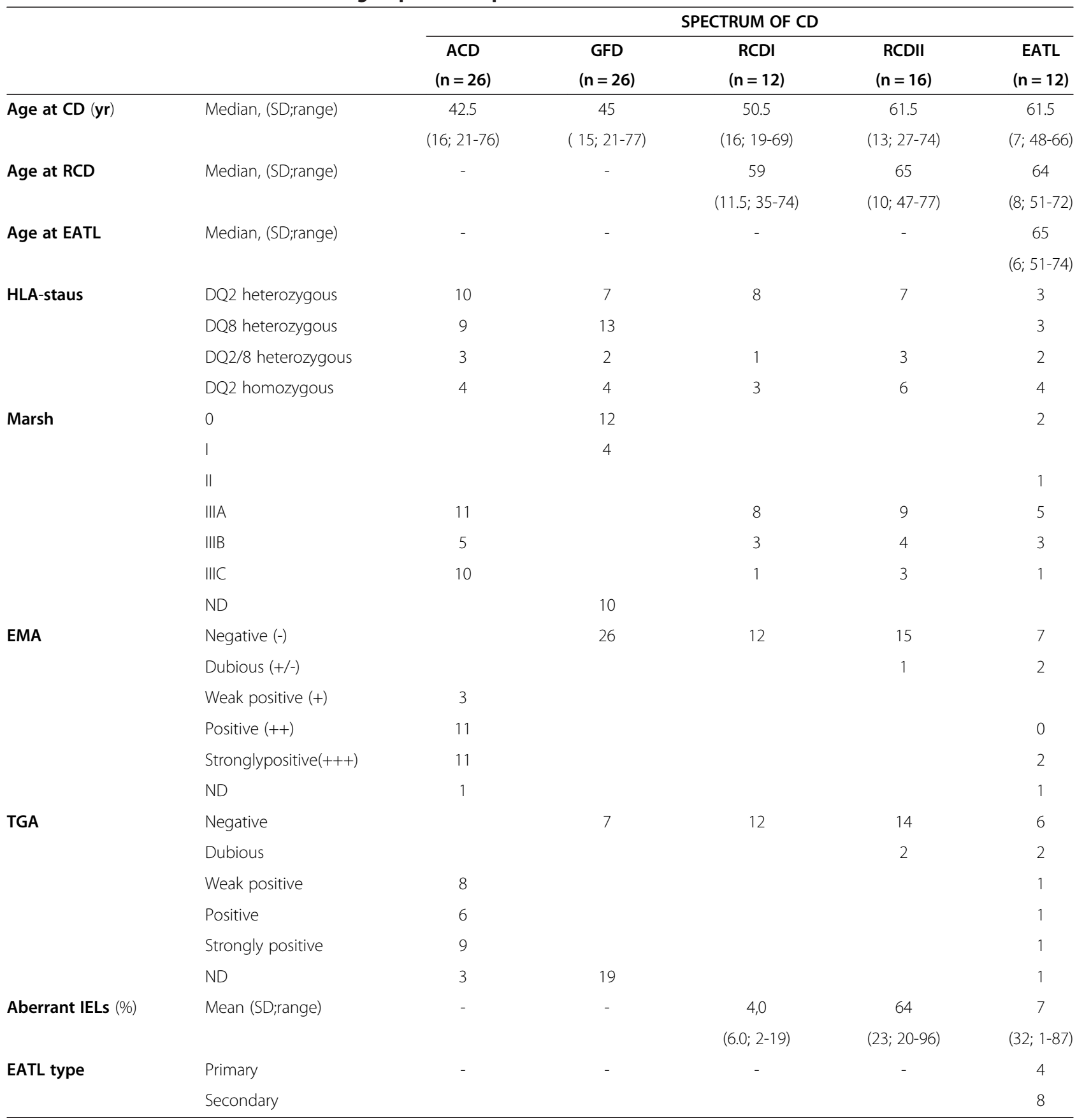

ACD: active coeliac disease patients; GFD: coeliac disease patients on a gluten-free diet; $R C D I$ and II: refractory coeliac disease type I and II; EATL: enteropathy associated T-cell lymphoma; EMA-A: endomysial antibodies; $t T G A-A$ : tissue transglutaminase antibodies; IELs: intra-epithelial lymphocytes.

analysis was to evaluate the ability of markers in peripheral blood to distinguish the various $\mathrm{CD}$ subsets. By doing so, it may also provide insight in the potential differences in underlying immunopathomechanisms of these disease subsets.

The results could be biased by cytokine production from different cellular sources in the peripheral blood, and therefore may not exactly mimic the intestinal inflammation [20]. However, other investigators have previously shown that blood cytokine profiles do reflect intestinal mRNA expression in active $C D$ patients [2,21-23]. Care must be taken in case our findings are extrapolated to other age groups, as it cannot be excluded that normal values vary over age. 


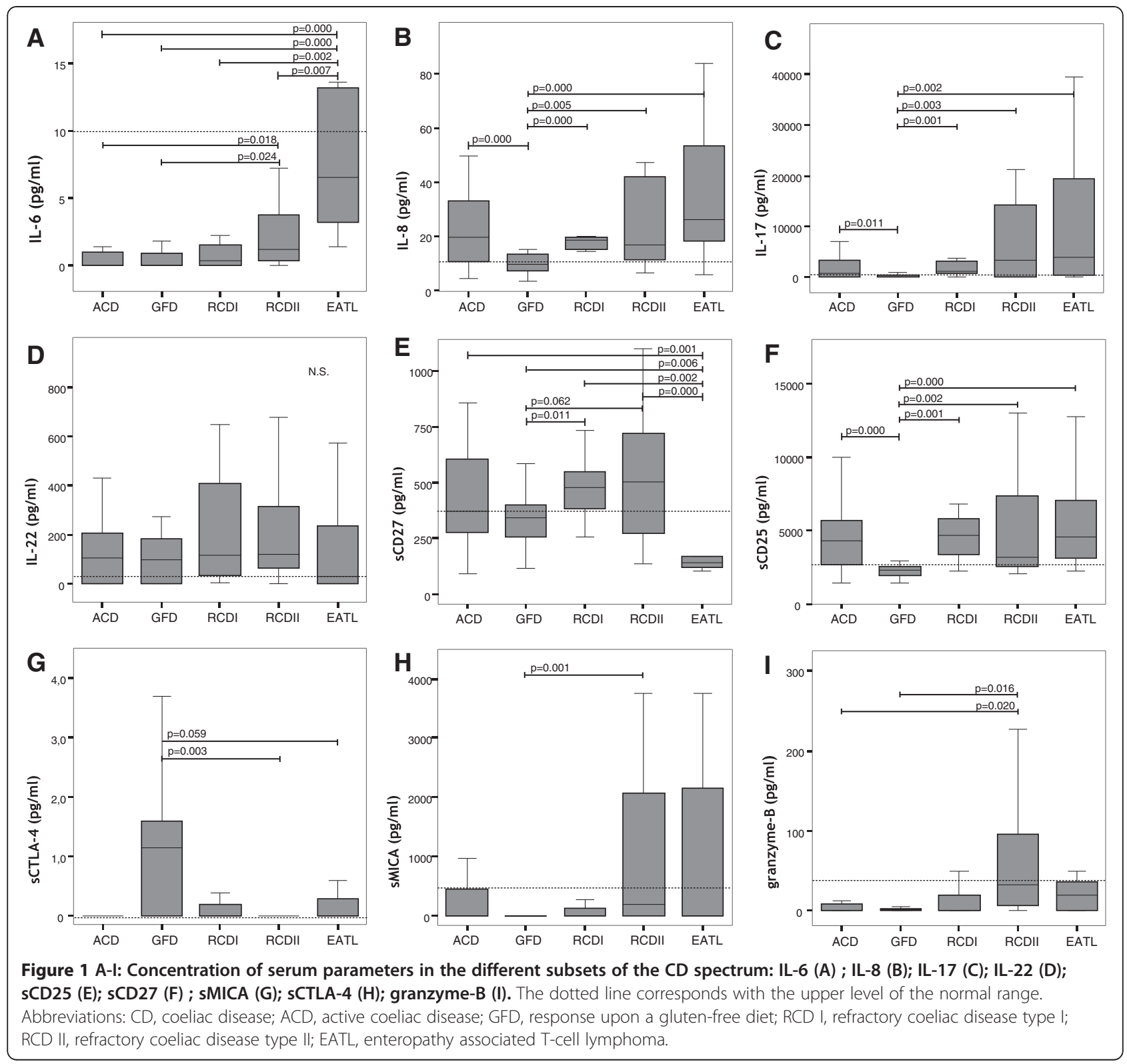

\section{Active $C D$ versus GFD}

Our results are in keeping with previous studies reporting on serum cytokine levels in ACD patients, that showed up-regulation of IL-8 [20,24], a chemokine produced by macrophages, epithelial as well as endothelial cells that attracts leukocytes to a site of inflammation. The same accounts for the observed elevated levels of sCD25, that is cleaved off from the membranous CD25 (IL-2R-alpha) during T-cell activation [25-27]. Furthermore, the increased levels of the pro-inflammatory cytokine IL-17 observed in the current study, supports the view that Th17 cells that produce this particular cytokine are involved in several auto-immune diseases and are suggested to have a pathogenic role in CD [3-5]. In contrast to the (pro-) inflammatory environmental characteristics observed in this analysis and to previous reports $[20,26,28]$, the levels of IL-6, illustrative of an acute phase response and a potent inducer of the Th17 pathway [29], were low and similar in both uncomplicated CD subsets. A clear explanation for these findings is lacking, especially as strongly increased levels were found in RCDII and EATL patients. Another cytokine involved in gut inflammation is IL-22 that is produced by Th17 and/or Th22 cells, where it is currently believed to exert regulatory functions [30,31]. Elevated levels of IL-22 have been found in the mucosa of Crohn's disease [32], but its role in CD is yet unclear [3,33]. Nevertheless, our results failed to reveal a correlation with IL-22 serum levels and disease activity or disease entity. sCD27 serum levels are increased in T-cell mediated 


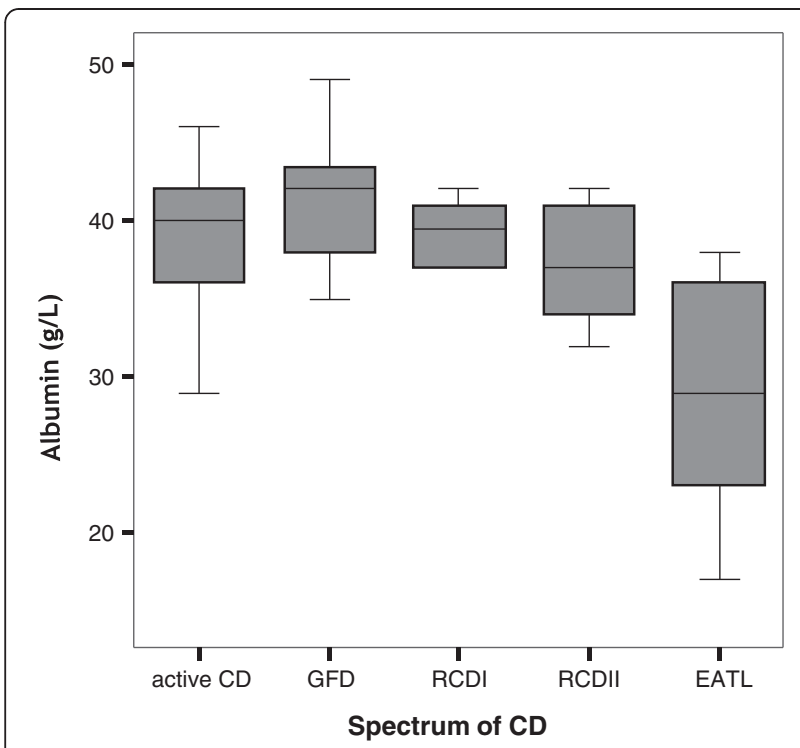

Figure 2 Concentration of albumin in the different subsets of the CD spectrum. The concentration of albumin is significantly higher in both the ACD $(p=0.003)$ and the GFD $(p=0.000)$ subset compared to the complicated forms of CD. In EATL patients clearly lower concentrations compared to both RCDI and II $(p=0.003$ and $p=0.037$, respectively) were observed. Abbreviations: $C D$, coeliac disease; ACD, active coeliac disease; GFD, response upon a gluten-free diet; RCD I, refractory coeliac disease type I; RCD II, refractory coeliac disease type II; EATL, enteropathy associated T-cell lymphoma.

diseases, including some auto-immune disorders [34,35]. However, the sCD27 levels were normal in ACD. This in contrast to the previously reported increased production of another lymphocyte activation marker sCD25. This dissimilarity is remarkable as in SLE patients SCD25 and sCD27 levels are strongly correlated during the whole disease course [36].

\section{The RCD complex}

Although, by general consent, RCD type I and II are considered two related disease entities within the spectrum of $C D$, it remains unclear if both diseases share a similar pathogenesis responsible for the glutenindependent inflammation. In keeping with the current opinion, our results showed in both types of RCD similar inflammatory characteristics and T-cell activation based on serum levels of IL-8, IL-17, IL-22 and sCD25, respectively. On the other hand, a transition from RCDI to RCDII has only been reported sporadically [16]. Theoretically, granzyme-B is a suited parameter to differentiate between RCD type I and II, since aberrant IELs are clearly cytotoxic and express high amounts of intracellular granzyme-B [12]. Although increased levels of soluble granzyme-B were observed in RCDII patients, these were not significantly higher than in RCDI patients.

\section{RCD versus uncomplicated $C D$}

Currently, it is unknown to what extent the glutenindependent inflammation as generally observed in RCD evolved from and/or differs from the gluten induced inflammation in active $\mathrm{CD}$. In this study, the proinflammatory T-cell response, including IL-8, IL-17, sCD25, in both types of RCD and ACD patients shows resemblance, with exception of evidently increased IL-6 levels in RCDII over active CD. This finding suggest a higher inflammatory state in RCDII than in ACD, however, similar levels of the inflammatory parameters CRP, ESR and leukocyte count were observed. In line with the lack of intestinal inflammation in patients adhering to a GFD, the pro-inflammatory response was significantly lower as compared to the complicated forms of $\mathrm{CD}$. Interestingly, in comparison to ACD patients, RCDII patients displayed a distinctive cytotoxic T-cell activation profile based on elevated serum levels of granzyme-B. The levels of these parameters observed in RCDI patients did not differ from either ACD or RCDII patients.

\section{Monitoring EATL development}

RCDII patients carry a high risk to develop an EATL, yet, so far no serum parameters for EATL development have been identified, including efforts in the present analysis. Based on the fact that EATL cells contain large amounts of granzyme-B [13], these levels were measured in the peripheral blood, but EATL patients did not contain higher levels than active CD or RCD patients. Furthermore, elevated levels of sCD27 [37] and sCD25 [38] have been suggested to be associated with tumour burden in some lymphoid neoplasia. Neoplastic lymphoid cells in non-Hodgkin lymphoma express CD27 and are considered responsible for the increased sCD27 production [37]. The current analysis failed to show increased sCD27 levels in EATL patients and thereby suggests that EATL regards a distinct type of lymphoma and does not aid in the identification of this particular lymphoma. In contrast to a previous study showing elevated sCD25 levels in EATL [39], we found comparable levels of sCD25 in EATL, RCDI-II and ACD. The same accounts for sMICA, that is cleaved from membranous MICA and has been shown to impair NKG2D mediated tumor surveillance in epithelial tumors [40], as well as it has shown potential as a prognostic parameter in hematopoietic malignancies since increased levels of sMICA were found in leukemia patients [41]. The only marker that distinguished EATL from all other groups was IL-6 and its levels correlated with CRP levels, indicating a more severe acute inflammatory response in EATL patients that is distinctive from the other subsets of CD. In accordance with our data, an association with 
IL-6 levels and survival in Hodgkin lymphoma has been recognized almost twenty years ago [42].

\section{Cytokine levels in comparison with other gastro-intestinal diseases}

Our data suggests that complicated CD is accompanied by a higher pro-inflammatory state as compared to uncomplicated CD. To provide insight in the extend of this inflammation, it can b compared to the cytokine profile in other (small) intestinal disease, such as Crohn's disease. Not only have elevated serum levels of IL-6 been reported in this disease, but these levels appear even higher than in complicated CD [43]. On the other hand, IL-6 and IL-8 serum levels in Helicobacter Pylori infected patients with peptic ulcer disease are not elevated [44].

\section{Clinical implication and potential application}

Taken together, for daily clinical practice the results of this analysis suggest that apart from detection of $C D$ associated antibodies and duodenal biopsies, other variables including IL-8, sCD25 and possibly IL-17, might be helpful in monitoring inflammatory disease status and differentiating between patients on a strict GFD and those diagnosed as having RCDI-II. In case CD specific antibodies remain mildly elevated, which is not rare in RCDI-II patients, serum levels of granzyme-B could possibly serve as an additional markers to distinguish $\mathrm{ACD}$ from RCDII, and will enable early intervention. However, the currently accepted diagnostic work-up of RCD remains required, pending prospective serum studies including larger series of patients.

\section{Conclusion}

In conclusion, both types of RCD are characterised by an ongoing and/or recurring inflammatory disease status showing great resemblance to that observed in $\mathrm{ACD}$ despite strict adherence to a GFD, yet differentiates itself by elevated serum IL- 6 concentrations in RCDII. Furthermore, in addition to this increased pro-inflammatory profile, RCDII reveals a distinctive cytotoxic T-cell activation profile as compared to $\mathrm{ACD}$ based on elevated levels of granzyme-B, whereas RCDI does not. Although no EATL-specific or -associated immunological parameters were found in this study, our ongoing efforts may identify relevant markers. Further research will also address the prospective and diagnostic value of the serum variables identified is this study in order to expand the clinical application of (R)CD serology beyond standard autoantibody testing.

\section{Abbreviations}

ACD: Active coeliac disease; CD: Coeliac disease; IEL: Intraepithelial lymphocyte; GFD: Gluten-free diet; RCD: Refractory coeliac disease; EATL: Enteropathy associated T-cell lymphoma.

\section{Competing interests}

Unrestricted grant from Astra Zeneca. Supported by the Coeliac Disease Consortium, The Netherlands. There is no competing interest.

\section{Authors' contributions}

GT conceived of the study, contributed to acquisition and interpretation of data and drafted the manuscript. RW carried out the ELISA and drafted the manuscript. BB and MS conceived of the study, and participated in its design and coordination and helped to draft the manuscript. HA and PB carried out the ELISA. VC performed the statistical analysis. CM participated in its design and coordination and helped to draft the manuscript. All authors read and approved the final manuscript.

\section{Author details}

${ }^{1}$ Department of Gastroenterology and Hepatology, VU University Medical Centre, PO Box 7057, Amsterdam 1007 MB, The Netherlands. Department of Pathology, VU University Medical Centre, Amsterdam, The Netherlands. ${ }^{3}$ Department of Epidemiology and Biostatistics, VU University Medical Centre, Amsterdam, The Netherlands. ${ }^{4}$ Department of Immunology, Erasmus MC, University Medical Centre, Rotterdam, The Netherlands.

Received: 21 May 2012 Accepted: 4 November 2012 Published: 12 November 2012

\section{References}

1. Jabri B, Sollid LM: Tissue-mediated control of immunopathology in coeliac disease. Nat Rev Immunol 2009, 9(12):858-870.

2. Nilsen EM, Jahnsen FL, Lundin KE, Johansen FE, Fausa O, Sollid LM, Jahnsen J, Scott H, Brandtzaeg P: Gluten induces an intestinal cytokine response strongly dominated by interferon gamma in patients with celiac disease. Gastroenterology 1998, 115(3):551-563.

3. Fernandez S, Molina IJ, Romero P, Gonzalez R, Pena J, Sanchez F, Reynoso FR, Perez-Navero JL, Estevez O, Ortega C, Santamaria M: Characterization of gliadin-specific Th17 cells from the mucosa of celiac disease patients. Am J Gastroenterol 2011, 106(3):528-538.

4. Monteleone I, Sarra M, Del Vecchio BG, Paoluzi OA, Franze E, Fina D, Fabrizi A, MacDonald TT, Pallone F, Monteleone G: Characterization of IL-17A-producing cells in celiac disease mucosa. J Immunol 2010, 184(4):2211-2218.

5. Sapone A, Lammers KM, Mazzarella G, Mikhailenko I, Carteni M, Casolaro V, Fasano A: Differential mucosal IL-17 expression in two gliadin-induced disorders: gluten sensitivity and the autoimmune enteropathy celiac disease. Int Arch Allergy Immunol 2010, 152(1):75-80.

6. Hue S, Mention JJ, Monteiro RC, Zhang S, Cellier C, Schmitz J, Verkarre V, Fodil N, Bahram S, Cerf-Bensussan N, Caillat-Zucman S: A direct role for NKG2D/MICA interaction in villous atrophy during celiac disease. Immunity 2004, 21(3):367-377.

7. Meresse B, Chen Z, Ciszewski C, Tretiakova M, Bhagat G, Krausz TN, Raulet DH, Lanier LL, Groh V, Spies T, Ebert EC, Green PH, Jabri B: Coordinated induction by IL15 of a TCR-independent NKG2D signaling pathway converts CTL into lymphokine-activated killer cells in celiac disease. Immunity 2004, 21(3):357-366.

8. Daum S, Cellier C, Mulder CJ: Refractory coeliac disease. Best Pract Res Clin Gastroenterol 2005, 19(3):413-424.

9. Rubio-Tapia A, Murray JA: Classification and management of refractory coeliac disease. Gut 2010, 59(4):547-557.

10. Verbeek WH, Goerres MS, von Blomberg BM, Oudejans JJ, Scholten PE, Hadithi M, Al-Toma A, Schreurs MW, Mulder CJ: Flow cytometric determination of aberrant intra-epithelial lymphocytes predicts T-cell lymphoma development more accurately than T-cell clonality analysis in Refractory Celiac Disease. Clin Immunol 2008, 126(1):48-56.

11. Cellier C, Patey N, Mauvieux L, Jabri B, Delabesse E, Cervoni JP, Burtin ML, Guy-Grand D, Bouhnik Y, Modigliani R, Barbier JP, Macintyre E, Brousse N, Cerf-Bensussan N: Abnormal intestinal intraepithelial lymphocytes in refractory sprue. Gastroenterology 1998, 114(3):471-481.

12. Tack GJ, van Wanrooij RL, Langerak AW, Tjon JM, von Blomberg BM, Heideman DA, van BJ, Koning F, Bouma G, Mulder CJ, Schreurs MW: Origin and immunophenotype of aberrant IEL in RCDII patients. Mol Immunol 2012, 50(4):262-270. 
13. de Bruin PC, Connolly CE, Oudejans JJ, Kummer JA, Jansen W, McCarthy CF, Meijer CJ: Enteropathy-associated T-cell lymphomas have a cytotoxic Tcell phenotype. Histopathology 1997, 31(4):313-317.

14. Al-Toma A, Verbeek WH, Hadithi M, von Blomberg BM, Mulder CJ: Survival in refractory coeliac disease and enteropathy-associated T-cell lymphoma: retrospective evaluation of single-centre experience. Gut 2007, 56(10):1373-1378.

15. Daum S, Ipczynski R, Schumann M, Wahnschaffe U, Zeitz M, Ullrich R: High rates of complications and substantial mortality in both types of refractory sprue. Eur J Gastroenterol Hepatol 2009, 21(1):66-70.

16. Malamut $G$, Afchain $P$, Verkarre $V$, Lecomte $T$, Amiot A, Damotte $D$, Bouhnik Y, Colombel JF, Delchier JC, Allez M, Cosnes J, Lavergne-Slove A, Meresse B, Trinquart L, Macintyre E, Radford-Weiss I, Hermine O, Brousse N, CerfBensussan N, Cellier C: Presentation and long-term follow-up of refractory celiac disease: comparison of type I with type II. Gastroenterology 2009, 136(1):81-90.

17. Rubio-Tapia A, Kelly DG, Lahr BD, Dogan A, Wu TT, Murray JA: Clinical staging and survival in refractory celiac disease: a single center experience. Gastroenterology 2009, 136(1):99-107.

18. Walker-Smith JA, Guandalini S, Schmitz J: Revised criteria for diagnosis of coeliac disease. Report of Working Group of European Society of Paediatric Gastroenterology and Nutrition. Arch Dis Child 1990, 65(8):909-911.

19. Oberhuber G, Granditsch G, Vogelsang H: The histopathology of coeliac disease: time for a standardized report scheme for pathologists. Eur J Gastroenterol Hepatol 1999, 11(10):1185-1194.

20. Manavalan JS, Hernandez L, Shah JG, Konikkara J, Naiyer AJ, Lee AR, Ciaccio E, Minaya MT, Green PH, Bhagat G: Serum cytokine elevations in celiac disease: association with disease presentation. Hum Immunol 2010, 71(1):50-57.

21. Kerttula TO, Hallstrom O, Maki M: Phenotypical characterization of peripheral blood T cells in patients with coeliac disease: elevation of antigen-primed CD45RO + T lymphocytes. Immunology 1995, 86(1):104-109.

22. Lahat N, Shapiro S, Karban A, Gerstein R, Kinarty A, Lerner A: Cytokine profile in coeliac disease. Scand J Immunol 1999, 49(4):441-446.

23. Nilsen EM, Lundin KE, Krajci P, Scott H, Sollid LM, Brandtzaeg P: Gluten specific, HLA-DQ restricted T cells from coeliac mucosa produce cytokines with Th1 or Th0 profile dominated by interferon gamma. Gut 1995, 37(6):766-776.

24. Hall RP III, Benbenisty KM, Mickle C, Takeuchi F, Streilein RD: Serum IL-8 in patients with dermatitis herpetiformis is produced in response to dietary gluten. J Invest Dermatol 2007, 127(9):2158-2165.

25. Blanco A, Garrote JA, Arranz E, Alonso M, Clavo C: Increased serum IL-2R levels in coeliac disease are related to CD4 but not CD8 antigens. J Pediatr Gastroenterol Nutr 1992, 15(4):413-417.

26. Romaldini CC, Barbieri D, Okay TS, Raiz R Jr, Cancado EL: Serum soluble interleukin-2 receptor, interleukin-6, and tumor necrosis factor-alpha levels in children with celiac disease: response to treatment. J Pediatr Gastroenterol Nutr 2002, 35(4):513-517.

27. Srivastava MD, Rossi TM, Lebenthal E: Serum soluble interleukin-2 receptor, soluble CD8 and soluble intercellular adhesion molecule-1 levels in Crohn's disease, celiac disease, and systemic lupus erythematosus. Res Commun Mol Pathol Pharmacol 1995, 87(1):21-26.

28. Fornari MC, Pedreira S, Niveloni S, Gonzalez D, Diez RA, Vazquez H, Mazure $R$, Sugai E, Smecuol E, Boerr L, Maurino E, Bai JC: Pre- and post-treatment serum levels of cytokines IL-1beta, IL- 6 , and IL-1 receptor antagonist in celiac disease. Are they related to the associated osteopenia? Am J Gastroenterol 1998, 93(3):413-418

29. Bettelli E, Carrier Y, Gao W, Korn T, Strom TB, Oukka M, Weiner HL, Kuchroo VK: Reciprocal developmental pathways for the generation of pathogenic effector TH17 and regulatory T cells. Nature 2006, 441(7090):235-238.

30. Strober W, Fuss IJ: Proinflammatory cytokines in the pathogenesis of inflammatory bowel diseases. Gastroenterology 2011, 140(6):1756-1767.

31. Trifari S, Kaplan CD, Tran EH, Crellin NK, Spits H: Identification of a human helper $\mathrm{T}$ cell population that has abundant production of interleukin 22 and is distinct from $\mathrm{T}(\mathrm{H})-17, \mathrm{~T}(\mathrm{H}) 1$ and $\mathrm{T}(\mathrm{H}) 2$ cells. Nat Immunol 2009, 10(8):864-871

32. Brand S, Beigel F, Olszak T, Zitzmann K, Eichhorst ST, Otte JM, Diepolder H, Marquardt A, Jagla W, Popp A, Leclair S, Herrmann K, Seiderer J,
Ochsenkuhn T, Goke B, Auernhammer CJ, Dambacher J: IL-22 is increased in active Crohn's disease and promotes proinflammatory gene expression and intestinal epithelial cell migration. Am J Physiol Gastrointest Liver Physiol 2006, 290(4):G827-G838.

33. Bodd M, Raki M, Tollefsen S, Fallang LE, Bergseng E, Lundin KE, Sollid LM: HLA-DQ2-restricted gluten-reactive T cells produce IL-21 but not IL-17 or IL-22. Mucosal Immunol 2010, 3(6):594-601.

34. Font J, Pallares L, Martorell J, Martinez E, Gaya A, Vives J, Ingelmo M: Elevated soluble CD27 levels in serum of patients with systemic lupus erythematosus. Clin Immunol Immunopathol 1996, 81(3):239-243.

35. Tak PP, Hintzen RQ, Teunissen JJ, Smeets TJ, Daha MR, van Lier RA, Kluin PM, Meinders AE, Swaak AJ, Breedveld FC: Expression of the activation antigen CD27 in rheumatoid arthritis. Clin Immunol Immunopathol 1996, 80(2):129-138.

36. Swaak AJ, Hintzen RQ, Huysen V, van den Brink HG, Smeenk JT: Serum levels of soluble forms of T cell activation antigens CD27 and CD25 in systemic lupus erythematosus in relation with lymphocytes count and disease course. Clin Rheumatol 1995, 14(3):293-300.

37. Metkar SS, Naresh KN, Manna PP, Srinivas V, Advani SH, Nadkarni JJ: Circulating levels of TNF alpha and TNF receptor superfamily members in lymphoid neoplasia. Am J Hematol 2000, 65(2):105-110.

38. Rubin LA, Nelson DL: The soluble interleukin-2 receptor: biology, function, and clinical application. Ann Intern Med 1990, 113(8):619-627.

39. Tack GJ, Verbeek WH, Van De Water JM, von Blomberg BM, Bhola SL, Ylstra B, Mulder CJ, Schreurs MW: Phenotypic and genomic analysis of an exceptional case of enteropathy associated T-cell lymphoma. Leuk Res 2010, 34(8):e183-e189.

40. Groh V, Wu J, Yee C, Spies T: Tumour-derived soluble MIC ligands impair expression of NKG2D and T-cell activation. Nature 2002, 419(6908):734-738.

41. Salih HR, Antropius H, Gieseke F, Lutz SZ, Kanz L, Rammensee HG, Steinle A: Functional expression and release of ligands for the activating immunoreceptor NKG2D in leukemia. Blood 2003, 102(4):1389-1396.

42. Kurzrock R, Redman J, Cabanillas F, Jones D, Rothberg J, Talpaz M: Serum interleukin 6 levels are elevated in lymphoma patients and correlate with survival in advanced Hodgkin's disease and with B symptoms. Cancer Res 1993, 53(9):2118-2122.

43. Gustot T, Lemmers A, Louis E, Nicaise C, Quertinmont E, Belaiche J, Roland S, Van GA, Deviere J, Franchimont D: Profile of soluble cytokine receptors in Crohn's disease. Gut 2005, 54(4):488-495.

44. Haghazali M, Molaei M, Mashayekhi R, Zojaji H, Pourhoseingholi MA, Shooshtarizadeh T, Mirsattari D, Zali MR: Proinflammatory cytokines and thrombomodulin in patients with peptic ulcer disease and gastric cancer, infected with Helicobacter pylori. Indian J Pathol Microbiol 2011, 54(1):103-106.

\section{doi:10.1186/1471-230X-12-159}

Cite this article as: Tack et al:: Serum parameters in the spectrum of coeliac disease: beyond standard antibody testing - a cohort study. BMC Gastroenterology 2012 12:159.

\section{Submit your next manuscript to BioMed Central and take full advantage of:}

- Convenient online submission

- Thorough peer review

- No space constraints or color figure charges

- Immediate publication on acceptance

- Inclusion in PubMed, CAS, Scopus and Google Scholar

- Research which is freely available for redistribution 